\title{
Les fondements de la responsabilité sociétale des entreprises et de sa communication
}

\section{Martine Bocquet}

\section{(2) OpenEdition}

1 Journals

Édition électronique

URL : http://journals.openedition.org/communicationorganisation/4768

DOI : 10.4000/communicationorganisation.4768

ISBN : 979-10-300-0155-6

ISSN : 1775-3546

Éditeur

Presses universitaires de Bordeaux

Édition imprimée

Date de publication : 1 décembre 2014

Pagination : 145-162

ISBN : 978-2-86781-905-6

ISSN : $1168-5549$

\section{Référence électronique}

Martine Bocquet, «Les fondements de la responsabilité sociétale des entreprises et de sa

communication », Communication et organisation [En ligne], 46 | 2014, mis en ligne le 01 décembre 2017, consulté le 26 avril 2019. URL : http://journals.openedition.org/

communicationorganisation/4768 ; DOI : 10.4000/communicationorganisation.4768 


\title{
Les fondements de la responsabilité sociétale des entreprises et de sa communication
}

\author{
Martine Bocquet ${ }^{1}$
}

Le discours sur la responsabilité sociale, ou plus largement, sociétale des entreprises (RSE) pratiqué par la plupart des grandes entreprises, qui s'est installé de plus en plus solidement au sein de la gestion comme de la communication institutionnelle, est considéré comme une innovation théorique et pratique des précédentes décennies. Il s'est, de toute évidence, établi dans la durée. Or, si l'on recherche le pourquoi de cet établissement et de ce début d'enracinement, on s'aperçoit qu'il s'agit de l'un des éléments de notre modernité qui entretient probablement des liens très anciens, jusqu'à l'époque médiévale. Qu'il s'agisse de l'enseignement des théologiens médiévaux, des montages canoniques normatifs associant droit romain et christianisme - aux racines de notre civilisation occidentale -, tout comme de la conduite des marchands et des artisans médiévaux et de la manière qu'ils avaient de gérer leurs affaires dans la cité, en application de ces normes et de ces préceptes. Il semblerait que ce soit ces problématiques anciennes qui, d'une certaine manière, resurgissent métamorphosées.

Au Moyen Âge, comme le rappelle Jacques Heers (1990), le marché était placé sous la protection des seigneurs et parfois des gens d'Église. Il se constituait à l'ombre des châteaux et des églises. Contrairement à ce que l'on pense généralement, le marché n'est pas né de l'ordre naturel des choses. La définition que donnait Max Weber ([1921]: 193,201) de la ville médiévale, en tant qu'organisation économique favorisant l'émergence de l'bomo aeconomicus, l'homme économique, par opposition à l'homo politicus de la cité antique où le citoyen restait d'abord un soldat et la cité une corporation de guerriers, paraît simplificatrice. Selon Jacques Heers et Henri Jorda $(2002: 104,122)$, la ville

\footnotetext{
1 Martine Bocquet est docteur en Sciences de l'Information et de la Communication. Ses recherches se concentrent sur les processus de communication liés à l'activité des entreprises et des institutions publiques. Elle est membre du CRPLC-UMR CNRS 8053. Elle a publié «Dynamiques sociosémiotiques : compréhension, processus, diachronie et fractales » dans la Revue Française des Sciences de l'Information et de la Communication $n^{\circ} 3 / 2013$. La SFSIC lui a décerné le $1^{\text {er }}$ Prix de thèse 2012/2013 en Sciences de l'Information et de la Communication. Elle est aussi membre de l'Association des diplômés du Celsa Paris IV-Sorbonne ; mm.bocquet@orange.fr
} 
médiévale a été une fondation politique avant d'être économique. Sa naissance a été encouragée par les seigneurs, sa place géographiquement déterminée. Les seigneurs ont assuré la protection des marchands qui s'y étaient installés. Le marché, lui aussi, n'a pas été une création spontanée. Dès le Haut Moyen Âge, les seigneurs et leurs armées ont suscité des trafics dans leurs places fortes. Ils ont attiré les commerçants et les artisans nécessaires à la vie et à l'entretien de la garnison. Cette activité économique, facteur de colonisation, a fixé des populations de plus en plus nombreuses et créé de véritables villes marchandes. Celles-ci n'étaient pas des fondations spontanées, mais tiraient leur origine d'un acte volontaire et politique, le plus souvent du seigneur laïc, parfois du seigneur ecclésiastique (Heers, [1990] : 49). Elles sont devenues ensuite, peu à peu, le lieu de l'organisation de la vie économique.

\section{Les prémisses médiévales de l'inscription de l'entreprise dans la cité}

\section{L'inscription des marchands et des artisans médiévaux dans la cité}

Lorsque l'on réfléchit aux origines de la RSE, l'exemple des corporations de métier médiévales, juridiquement très structurées, saute aux yeux. Elles se chargeaient dans les villes de nombreuses tâches civiques, que nous appellerions aujourd'hui de responsabilité sociétale. Ces communautés restreintes ou partielles jouaient, à l'époque médiévale, un rôle économique et social non négligeable, selon Régine Pernoud (1992: 65, 71)2 , et beaucoup plus large que l'exercice du simple monopole de la profession. Les corporations et les confréries s'occupaient de régulations internes, de la police de la profession, de la formation des compagnons et des maîtres, de contrôles exercés par les Prud'hommes jurés, des freins mis à ce que nous appellerions de nos jours la concentration verticale. Constituées en réseaux d'artisans mais aussi parfois de marchands, elles garantissaient une marque de qualité individuelle pour l'artisan ou collective pour la corporation, à chaque étape du processus de fabrication, qui était tracé, ainsi qu'un contrôle des prix et des conditions de travail, des aides et des secours mutuels en cas de maladie ou de décès. Elles se chargeaient, tout comme le faisaient les marchands eux-mêmes, de tâches d'intérêt commun pour le compte de la cité que nous traduirions de nos jours sous les termes de mécénat d'entreprise, de fondation ou de responsabilité sociétale, etc : la fondation d'hospices, la construction et l'entretien des remparts, des halles, le guet, etc. Certaines de celles-ci apparaissent voisines de celles dévolues de nos jours aux Chambres consulaires ou Chambres de commerce, qui entretiennent les ports, les aéroports, récoltent des taxes, ont une mission importante de formation, d'aide à la création d'entreprises et à l'innovation, etc. Ce qui domine dans ces corporations médiévales, selon Régine Pernoud (1992: 74), c'est le souci du bien commun, selon le principe

2 Cf. également Jacques Le Goff, 2010 : 126 ; Jacques Le Goff, [1956]; Baschet, 2006 : 198. 
- rappelé par les théologiens médiévaux (Brants, 1895 ; Braid, 2011 ; Jacques Le Goff, 2010 : 114) - que toute besogne doit être faite pour le bien commun, même s'il est tout à fait admis de s'enrichir. Ce qui compte, c'est l'emploi que l'on fait de cette richesse, sous la condition de ne pas la garder pour soi seul, selon le péché d'avarice, mais aussi, de façon équitable - mais non égalitaire -, de savoir en faire profiter les proches et les gens de la cité, selon le principe d'une économie bonne et loyale.

Danièle Courtemanche et Myriam Chopin-Pagotto (2002) ont étudié les chroniques, quasiment biographiques de deux riches marchands drapiers, Etienne de Médicis (circa 1475-1565)33, bourgeois du Puy, et Philippe de Vigneulles (1471-circa 1528)4, bourgeois de Metz. Tous les deux témoignent de leur engagement au sein de leur ville, et s'efforcent d'en transmettre la mémoire. Ils témoignent du rapport qui s'opère, au XVI siècle, entre ces marchands de renom, leur ville et la construction par écrit d'une mémoire urbaine. Ils s'efforcent de mettre en garde les citoyens et les générations à venir contre toute action qui conduirait à l'éclatement de la cohésion civique, sociale et religieuse. Ils se font les gardiens de l'idéologie du bon gouvernement de leur ville. Ce ne sont pas des historiographes officiels, mais avant tout des marchands qui ont fait fortune dans leur ville et qui, grâce à celle-ci et à sa cohésion, ont pu être liés aux divers réseaux politiques, économiques et culturels. Ils suivent un chemin assez identique aux chroniqueurs italiens, notamment les marchands toscans du XIV e siècle (Bec, 1984: 48), dont les écrits, partagés entre réflexion personnelle et enregistrement des événements qui touchent la cité, se faisaient volontiers pédagogues, condamnant les mauvais citoyens et trouvant prétexte pour des leçons pratiques de comportement politique, toutes faites de prudence et de respect des autorités urbaines. Pour les chroniqueurs italiens, comme pour Etienne de Médicis et Philippe de Vigneulles, la construction de la mémoire de leur ville fait partie d'un projet pédagogique. Ils apparaissent comme les représentants du civisme marchand, que l'on observe à l'époque, défenseurs de la pleine maturité des institutions urbaines.

\section{Les fondements romano-canoniques de la RSE}

On assigne souvent à la responsabilité sociétale de l'entreprise un ancrage dans la pensée religieuse nord-américaine, quoiqu'il faille aussi considérer son émergence dans les idées de la démocratie sociale chrétienne européenne. Néanmoins, Joan Le Goff (2010) a montré que l'on devait remonter plus loin dans le temps, à l'époque médiévale. En recherchant les circonstances de l'affirmation de cette notion dans son contexte et les connexions idéelles qui ont présidé à son émergence, il s'est aperçu que celles-ci procédaient d'un

3 Auteur du livre de Podio ou Chroniques [de la ville du Puy], circa 1500-1562.

4 Auteur de la Chronique de Metz, 1491-1525. 
apport en profondeur excédant la notion elle-même et se répercutant dans l'ensemble des pratiques et des discours managériaux, comme des techniques. La conception même du management occidental se serait construite sur un substrat religieux commun, à partir d'un système de pensée élaboré, complexe et contraignant, que celui-ci s'est approprié, tout comme l'ont fait les sciences de gestion. Pierre Legendre $(1999,2009)$ a montré qu'il fallait remonter jusqu'au moment, aux XII ${ }^{e}$ et XIII ${ }^{e}$ siècles, où les institutions chrétiennes ont absorbé le droit romain pour en faire un système normatif cohérent, désigné sous le terme de romano-canonique, qui constitua un socle normatif puissant.

Le droit romain, fondu dans le droit canonique chrétien, procura à l'Église une grande efficience politique et sociale (Legendre, $2009: 369,380$ ). Cette conception de la norme fut suffisamment souple pour se maintenir, une fois laïcisée, car elle n'était pas basée sur des règles sociales - absentes dans les Évangiles -, mais sur une sorte de vide de la forme évangélique originale - qui ne contenait que des principes. Ce vide originel a été comblé par un construit romano-canonique modifiable lentement. En retour, cette manière abstraite de penser la norme, bâtie sur des principes considérés comme universels, a été soutenue par une grande propension à la technicité, appelée par nécessité à remplir le vide initial. On retrouve cette technicité dans beaucoup de domaines, depuis le droit, la philosophie, la logique et la sémiotique médiévales jusqu'à l'architecture. Ce primat de la technicité, remodelant sans cesse la forme abstraite de la norme et la remplissant, a ouvert la voie à une conception techniciste et instrumentaliste des normes, intrinsèquement anhistorique. Cela s'est produit alors même que l'Église et les théologiens étaient les seuls instances capables de penser l'échange, le travail, la production, la vente, le profit, le juste salaire, etc, leur donnant un sens éthique et juridique, au sens d'une économie bonne et loyale; selon une pensée que nous qualifierions aujourd'hui d'économique et de gestionnaire, mais basée sur l'idée chrétienne de Rédemption et de Salut, avec ses représentations, ses symboles, sa liturgie et ses rituels, conduisant au respect et à la légitimation de l'autorité. Il s'agissait d'une légitimation de l'autorité faite aussi de surveillance du respect des règles, de jugement et de sanctions, du moins morales, articulant l'individuel et le collectif. Une sorte d'oubli de ces origines s'est ensuite emparée des sciences de gestion, qui ne se conçoivent que dans cet oubli, de façon anhistorique (Legendre, 1982). Cette amnésie délibérée empêche le discours gestionnaire de percevoir le substrat idéologique originel, lequel agit pourtant souvent de façon quasi inconsciente, à la fois sur les producteurs et sur les récepteurs. Selon Pierre Legendre (2005, 2009), ce discours s'est servi d'un subterfuge à la référence indicible chrétienne et ecclésiale. Ce subterfuge s'est bâti autour de la logique économique de l'efficacité, à l'aide d'une écriture technique et scientifique développant le concept du management, lequel a aussi irradié la sphère des institutions publiques et de l'État. Ce discours s'est construit en s'aidant d'un juridisme très technique - hérité lui aussi du droit romain et 
de la même source romano-canonique -, qui était bâti sur l'enchaînement des causalités et l'art scholastique des casuistiques. L'héritage scientifique de ce modèle a fini par le rendre indiscutable. Joan Le Goff (2010 : 278) explique que le texte premier théologique, une fois institutionnalisé, a été marqué par un lexique technique fondé sur une logique de l'efficacité, donnée comme échappant à l'analyse, donc immanente et incontestable, car réelle et objective, au sens d'une loi scientifique muée en loi naturelle. Cette quête de l'efficacité conduit à un absolu moral et à une doctrine fondée sur des valeurs impératives, parce qu'énoncées comme des vérités scientifiques. Ce glissement vers la morale expliquerait pourquoi le modèle gestionnaire irradie tant de nos jours la vie sociale dans son ensemble.

Ce serait cet impératif moral, fondé sur un management efficace - «efficace car moral et moral parce qu'efficace " (Joan Le Goff, 2010 : 282) -, que nous retrouvons aujourd'hui dans la responsabilité sociétale des entreprises. Ainsi laïcisées, l'éthique et la morale sont ici mises au service d'un bon gouvernement des entreprises, placé sous l'impératif dogmatique de la gestion. Il ne s'agit pas d'une question de conviction religieuse, mais d'un caractère bienveillant qui s'exprime dans les idées de prospérité, de satisfaction du bien-être, de paix sociale, héritées d'un entrelacs entre provenance idéelle du christianisme et du droit romain, revêtu d'un habit scientifique exprimant rigueur, progrès, vérité, etc, et permettant une validation de la croyance.

Pour que cela s'impose, il a fallu à la gestion une compétence double, c'està-dire un savoir-faire, à la fois discursif - communicationnel - et normatif. Ce serait l'une des clefs de la réussite actuelle de la responsabilité sociétale des entreprises d'avoir su se placer en médiateur de ce couple, notamment par l'insistance mise sur la promesse, de caractère scientifique, d'un avenir meilleur au sens matériel comme moral. Ainsi se déploie une nouvelle dimension de la matrice originelle de la pensée gestionnaire et de son autorité, alliant concept de gestion et communication. La responsabilité sociétale des entreprises, alternative aux impasses d'une visée purement utilitariste, devient dès lors un outil stratégique ayant pour visée une extension du champ de la performance des firmes à des variables autres que financières, sans cependant exclure celles-ci, étendant le champ d'influence de l'entreprise au-delà de ses frontières propres et investissant l'espace public. Fondée sur une éthique universelle, à l'image de l'éthique chrétienne, la responsabilité sociétale des entreprises s'est dotée d'un cadre imparable, basé sur le glissement vers la morale, qui a toujours caractérisé la pensée gestionnaire dans son ensemble. $\hat{A}$ ce titre, les sciences de gestion se font communicationnelles et productrices d'images, telles le consommateur responsable, le dirigeant responsable etc. Joan Le Goff (2010 : 286) y voit l'expression d'une idéologie transmise par des symboles, des rites et des croyances, selon un appareil théologique et universel, à l'image de l'appareil théologique chrétien, renforçant la crédibilité des objectifs traditionnels de la direction d'entreprise et fondant la 
légitimité du discours gestionnaire, en l'inscrivant sur le terrain des valeurs et de la durée. Ce discours revêt les caractéristiques d'un nouvel encastrement de l'entreprise dans la cité, avec toutes ses connotations de sécurité, de communauté, de lien social, de normativité quasi religieuse, de régulation et de liberté limitée, comme de contrôles normatifs imposés au nom de valeurs à défendre, ne connaissant de frontières ni matérielles ni juridiques, au nom d'une transparence se transformant peu à peu en surveillance sous l'égide du collectif. Les travaux de Joan Le Goff sonnent, sur ce point, comme une mise en garde. En sorte que la RSE rassure, apparaît nécessaire, mais peut aussi inquiéter de par ces dérives potentielles intrinsèques.

\section{Lien contractuel, norme institutionnelle ou relation communautaire?}

\section{Les fondements}

Lorsque, de nos jours, nous examinons la notion de RSE, différentes conceptions se présentent, voire s'opposent. Une conception contractualiste, une conception institutionnaliste - laquelle peut être liée à une conception normative - et une conception communautaire. Se retrouve ici la dichotomie essentielle entre le lien et la norme. Le lien est de nature proprement contractuel, du simple contrat au contrat social. La norme, qu'elle se décline sous la forme de coutumes, d'usages, d'habitus, est de l'ordre du status, du statut et de l'institutionnel. Alain Supiot (2007) a rappelé cette distinction fondamentale dans les rapports de type juridique. Dès lors que les concepts de responsabilité sociale ou sociétale et de développement durable nous font aborder le champ institutionnel, donc des problématiques communicationnelles intéressant le politique, le juridique resurgit.

Il faut en conséquence aborder la distinction entre le lien et la norme, car elle a des implications dans le discours institutionnel. Dans la culture juridique occidentale, la lex, la loi, désignait à l'origine, dans son sens religieux premier, un impératif s'imposant à tous. Cette hétéronomie imposait la norme comme une structure qui, à la manière d'une langue, transcende les individus et les générations, leur impose le respect d'un sens commun et rend possible l'expression de leurs accords et de leurs désaccords. La norme convoque le statut et l'institution : le souverain. Tandis que le contrat, s'il met en jeu des statuts, tend à déconstruire ceux-ci au profit du lien, d'un lien contractuel marqué par une certaine autonomie : le droit devient celui que les parties ont conclu entre elles, il fait la loi des parties et convoque le suzerain. Le contrat, comme nous allons le voir, est à l'origine de la féodalité, entendue comme lien contractuel de vassal à suzerain, incluant ses propres ramifications normatives.

Certains auteurs, Marc Bloch (1931 : 6), Jacques Foviaux (1986 : 284, 333, 353), Régine Pernoud (1992 : 141) ont montré que les sociétés mérovingienne et carolingienne, puis la société féodale, avaient fonctionné 
selon un mode particulièrement contractualiste. Marc Bloch relevait que l'hommage vassalique, qui lui apparaissait sous la forme d'un contrat, et la vassalité avaient contribué en Europe à répandre dans les esprits la notion obscure, mais vigoureuse, d'une sorte de contrat social. Jacques Foviaux a montré l'aspect pré-contractualiste de la société mérovingienne, fondée sur le système de la commendatio - l'acte de se recommander à la protection d'un seigneur en échange de services -, ancêtre de l'hommage vassalique, au point de qualifier la monarchie mérovingienne de monarchie contractuelle. Selon Régine Pernoud, la société féodale était faite, toute entière, d'une multitude de contrats privés reliant entre eux les individus et les familles, les feux, à tous les échelons de la société, faisant de la société féodale une société contractuelle.

De façon antinomique, les légistes royaux ont développé, à partir du $\mathrm{XIII}^{\mathrm{e}}$ siècle, le concept de puissance publique en tant que postulat juridique, sans contrat possible pour être établi. De leur côté, les théologiens élaboraient la synthèse de l'enseignement d'Aristote et de la théologie chrétienne, établissant que la vie en commun était une nécessité de la vie humaine, de façon non contractuelle. Pour les théologiens, le principe de l'autorité se trouvait dans le peuple - et non dans le Prince -, insistant sur le caractère collectif de l'État, donnant ainsi un fondement moral à l'instinct social ; mais en ce sens que la vox populi est la voix de Dieu, vox Dei, et que toute autorité agit ainsi par délégation divine. Ils ne se souciaient point des modalités matérielles d'attribution de l'autorité. Ces deux positions, des légistes et des théologiens, somme toute institutionnelles, qu'il est possible de désigner d'une part de puissance publique et d'autre part de communautaire, en fait, se complétaient, par opposition au pacte mutuel féodal, de nature contractuelle.

De façon presque anachronique, Jean-Jacques Rousseau allait au

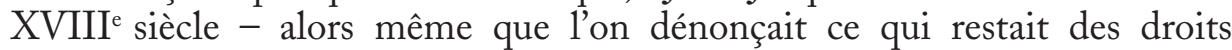
féodaux - redécouvrir la notion de contrat, placé de façon utopique à l'origine de la société, en tant que contrat collectif et non plus personnel, mais restant volontaire, résultant des progrès de la civilisation. Ce pacte social fondait le citoyen, la propriété devenant à la fois privée et publique. Dans cette définition, la distinction, entre public et privé, péniblement élaborée par les scholiastes médiévaux et fort peu pratiquée, s'estompait à nouveau quelque peu.

De la même manière, le contrat, retravaillé par les scholiastes médiévaux, avait évolué d'un aspect féodal, basé sur le consentement mutuel et le serment, vers une forme médiévale (non féodale) excluant le serment aux connotations païennes, qui deviendra celle du Code Civil de 1802 : l'échange de volonté par consentement mutuel entre personnes réputées égales. Cette conception de nature libérale avait, au départ, été fondée sur une conception canoniste du contrat, mettant en avant la morale chrétienne et les obligations du chrétien dans la parole tenue, sans qu'il soit nécessaire de les formuler par un serment ou des actes, pour évoluer vers la conception laïque et abstraite d'un simple 
échange de consentement entre personnes réputées, par abstraction, égales en droit (Supiot, 2000). Cette forme libérale du contrat allait irradier le monde contemporain.

\section{L'évolution}

Mais, de façon hybride, la dialectique contractuelle a fait du contrat contemporain la source d'obligations de nature normative, pas seulement entre les parties, mais prenant aussi valeur réglementaire pour d'autres individus qui n'y étaient pas partie et leurs successeurs. Il en est ainsi du droit des conventions collectives, de la sécurité sociale, etc, d'une grande partie du Droit social, retirant à l'État une aussi grande partie de sa fonction normative, tout comme au parlement. Lidéologie contractualiste imprègne le néo-libéralisme contemporain et la mondialisation des années 1990 et 2000, devenant la culture du management. Elle imprègne aussi les structures du monde économique, faites de réseaux contractuels de groupes prenant la forme de holdings, de filiales, de franchisés et de sous-traitants, pris dans les mailles de phénomènes d'allégeances nouvelles et de normes de nature contractuelle. Alain Supiot $(2000,2009$ : 174, 194) qualifie ce phénomène de reféodalisation par le contrat.

Le souverain régit, plus ou moins directement, tandis que le suzerain, qui n'a plus de contrôle qu'indirect sur ses vassaux, régule toujours de façon distante et indirecte, même si cette forme contractuelle peut placer les gens dans des liens de dépendance contraignants, car il n'existe pas de forme contractuelle où l'égalité des parties soit réelle. Ainsi, le contrat crée ses propres normes, selon un principe de subsidiarité. Alain Supiot (2007 : 31) observe que, derrière l'idéologie contractualiste de la responsabilité sociale ou sociétale des entreprises, se profile ce retour au féodal. Il faut se défaire des illusions du tout contractuel. "[...] le contractualisme porte aussi en lui les germes de son propre dépassement, ou plus exactement il ne constitue que la face la plus visible et la plus inquiétante du processus de contractualisation du Droit. Un regard attentif sur le droit contemporain laisse entrevoir une autre face de ce processus : celle de la résurgence des structures juridiques féodales. ».

\section{Encastrement de l'économique ou libéralisme économique}

Nicolas Postel et Richard Sobel (2010 : 3), citant Karl Polanyi ([1944]), rappellent que dans les sociétés pré-industrielles, donc à l'époque médiévale, le domaine économique était confiné dans un statut social, finalement subordonné au système des besoins. Ces activités se déployaient toujours sous une forme sociale encadrée, maîtrisée et régulée par des institutions d'ordre juridique ou politique ; pour l'éthique et la morale : l'Église; pour les régulations économiques : principalement le seigneur laïc ou ecclésiastique, ainsi que le conseil municipal et les corporations, mais aussi dans les villes royales le Roi ou son procureur. La société médiévale - comme la société de 
l'Ancien Régime, dans une moindre mesure cependant - avait mis en place des dispositifs religieux, juridiques et sociaux complexes, qui, en tant que normes, avaient pour conséquence que les pratiques économiques ne pouvaient former une totalité marchande homogène, susceptible d'extraversion et qui serait imperméable aux régulations socio-politiques comme morales (Braudel, 1979) ; c'est ce que Alain Supiot (2000 : 8) nomme le Garant des Pactes.

À partir de la chute de l'Ancien Régime, on a assisté, au nom de la liberté, à l'émergence du primat d'une conception contractualiste des échanges et de l'économie, qui allait s'étendre à l'entreprise, selon une visée de plus en plus utilitariste. Karl Polanyi ([1944]) a montré que, dans le sillage de la révolution industrielle, le monde économique s'est peu à peu désencastré de la gangue régulatrice pré-industrielle, à partir de la fiction de la fonction autorégulatrice du marché, théorisée par les économistes classiques et néo-classiques. Le marché s'autorégulerait par la confrontation entre les flux anonymes, contractuels, de l'offre et de la demande, selon un modèle proche de celui du marché de concurrence pure et parfaite; donc en l'absence de tout Garant des Pactes. Ce ne fut naturellement jamais qu'une fiction. Cette autonomisation de la sphère économique ne fut jamais totale, parce qu'aucun marché ne peut exister indépendamment de la société qui le crée. Le marché s'avère ne pas être une réalité naturelle sui generis. Ce sont forcément, toujours, des dispositifs politico-juridiques particuliers qui constituent les conditions de la possibilité du marché.

Le chercheur américain R. Edward Freeman (1984) a développé une théorie libérale de la RSE à travers sa théorie des parties prenantes. Celleci se caractérise par la disparition d'une médiation collective entre acteurs, et en particulier par la disparition de toute forme étatique. Les individus, ayant pris conscience de leurs capacités éthiques, pourraient s'auto-organiser au sein d'accords volontaires, parce que toutes les transactions impliquent des clients, des fournisseurs, des employés et des financiers et que les parties prenantes acceptent leurs responsabilités et reconnaissent des valeurs. La concurrence serait contrebalancée par la recherche permanente d'accords entre contractants, sur le modèle des relations entre entreprises, au sein d'un système de responsabilité individuelle et de négociations. Le marché ne serait plus auto-régulé, mais régulé par les contractants eux-mêmes et les parties prenantes, sur le mode contractuel.

Comme nous l'avons vu, Nicolas Postel et Richard Sobel (2010:14) pensent que l'économie est un problème structurel très en amont de la négociation marchande, qui pose le problème de l'articulation de la logique sociale éthicopolitique et de la logique productive instrumentale. C'est un problème de désencastrement de l'économique, imposant une logique destructrice par la marchandisation des piliers de la société, que des contrats inter-individuels ne peuvent résoudre. Ils en reviennent à une ré-institutionnalisation de 
l'économique imposant des règles collectives, tout autant que des conventions communes, le mode contractuel ne pouvant être complètement écarté.

Si la RSE est appréhendée dans la visée d'une gestion durable du travail et des ressources environnementales, elle peut être aussi considérée dans une perspective institutionnaliste et s'annoncer comme une nouvelle forme de réencastrement de l'économique, favorisé par cette institutionnalisation. C'est pourtant à une vision contractualiste, qu'est souvent étroitement associée la RSE, portée par le retour au tout contractuel, selon la théorie défendue par R. Edward Freeman (1984) d'une éthique contractuelle. La communication institutionnelle se situe à cette croisée des chemins, entre contractuel abstrait et institutionnel, peut-être plus concret, entre abstraction généralisée et réel, recherchant une transcendance tacitement réclamée, laquelle se décline déjà sous différentes formes.

\section{La construction d'un nouvel espace socio-politique par le discours et les pratiques de la RSE}

Depuis plusieurs décennies, nous assistons donc à une institutionnalisation de l'entreprise, véhiculée par sa communication et curieusement renforcée par une vision contractualiste de la responsabilité sociale ou sociétale, créatrice de normes voulues universelles et autorégulatrices. Face à cette institutionnalisation de l'entreprise, peut être stigmatisée une érosion de la sphère publique, du fait d'une désinstitutionnalisation de l'État et des institutions publiques. Ceci paraît concourir à l'apparition d'un nouvel espace socio-politique.

Selon Dominique Wolton (2007 : 41), dans une société ouverte, à côté du problème essentiel de l'équilibre des pouvoirs, se pose, avec la socialisation de toute activité de la société, la question de la place, du rôle et de la limite des différents discours. Thierry Libaert (2006) note que, sous l'effet conjugué d'un relatif désengagement de l'État et d'un accroissement de l'opinion publique, l'entreprise a accru le périmètre de sa prise de parole. Dès lors que l'entreprise est de plus en plus appréhendée comme une institution, elle se voit transférer de fait par les pratiques, mais aussi souvent de droit, par la législation et la réglementation, une grande partie du discours citoyen basé sur le développement durable, le comportement civique, écologique... Ce discours citoyen se fait, dès lors, porteur d'engagements et de promesses dans le cadre de la RSE.

Jacques Viers et Vincent Brulois (2009) se demandent, eux aussi, si l'entreprise, devenue institution, contrainte parfois à se substituer à des pouvoirs publics défaillants, ne crée pas un nouvel espace socio-politique. N'a-t-elle pas une propension à devenir une institution empiétant sur le territoire de l'État? Ils relèvent une désinstitutionnalisation de l'État, c'est-àdire un retrait de celui-ci, entraînant par contrecoup une institutionnalisation de l'entreprise, échafaudée autour de la responsabilité sociale ou sociétale de 
l'entreprise et de sa communication. Les deux auteurs relèvent, par ailleurs, que de grandes entreprises européennes concluent, avec les fédérations syndicales internationales de leur secteur, des accords-cadres internationaux étendus à tout le groupe, relatifs à leur responsabilité sociale ou sociétale, débordant largement le cadre étatique. Ils voient dans ces accords, qui portent sur tous les aspects de la RSE, y compris les questions environnementales, une petite révolution dans le dialogue social traditionnel. Nous assistons, et la liste n'est pas exhaustive, à l'entrée de nouveaux acteurs dans l'entreprise, comme les ONG - certaines entreprises créent des partenariats avec cellesci -, à la création de nouvelle scène de délibération que sont les comités des parties prenantes, à la mise en place de nouvelles formes de régulation dans le domaine des ressources humaines, que sont les accords-cadres internationaux relatifs à la RSE - portant sur les droits de l'homme et de l'environnement -, à de nouveaux éléments de négociation, dans ce que l'on désigne par le reporting $R S E$ et la mise en place d'indicateurs RSE à l'échelle internationale, etc. L'entreprise crée, de cette façon, son propre espace public, comme le pressentait Jürgen Habermas ([1962]). Les institutions démocratiques de l'État paraissent devenir une partie prenante parmi d'autres. L'entreprise rend des comptes à la société, quasiment à son rythme, et un peu comme elle l'entend. Elle choisit volontairement d'adhérer à des chartes ou d'adopter des référentiels, mais a tendance à refuser toute règle internationale contraignante.

L'entreprise crée son espace public en sélectionnant ses parties prenantes, donc en ignorant les autres, l'État devenant ainsi une partie prenante parmi d'autres. Elle ne rend compte qu'à la société qu'elle s'est ainsi forgée, celle qui figure dans son espace public, lequel ne peut toujours qu'être délimité, fini. Lorsqu'elle se trouve face à des États faibles, elle crée ses propres règles en négociant avec eux, créant un droit du contrat au détriment du droit local. Selon Laurent Habib (2010 : 42), faute de pouvoir s'appuyer sur un appareil d'État fiable et réactif, le public effectue une projection de l'exécutif étatique vers l'entreprise. On attend de cette dernière qu'elle aille au-delà de son rôle premier de productrice de richesses matérielles ou immatérielles, et qu'elle incarne un rôle citoyen à la place du monde politique. Ce type de communication des entreprises, imprégné des thèmes liés à la responsabilité sociétale, encourage les individus à rêver l'impossible, c'est-àdire que l'entreprise prenne en charge une forme de régulation sociale ou plus largement sociétale.

\section{Un retour vers la relation communautaire : une résurgence ancienne}

Nicole d'Almeida ([2001] : 212, 217) oppose, au mode contractuel, le mode communautaire. L'approche communautaire, qui est ancienne, envisage la relation de travail comme une relation d'appartenance et non comme une relation abstraite d'échanges régie par un contrat. Elle suppose une 
communauté de vie, des préoccupations d'intérêts communs, l'engagement et une certaine unité de ses parties.

L'entreprise, vécue en tant que communauté, renvoie à une réalité historique que l'on croyait révolue, dont la corporation médiévale, comme nous l'avons vu, est l'ancêtre. Elle provient de l'ère préindustrielle, marquée par l'organisation en métier et par la société de corps de l'Ancien Régime. Il s'agissait d'une dynamique de l'apprentissage et de l'excellence. Ce modèle commensal reposait sur la proximité - exprimée par l'étymologie du terme, qui est le partage symbolique du repas - unissant artisans, compagnons et apprentis. Ce modèle se serait transformé, au cours de la révolution industrielle, pour prendre les traits du modèle paternaliste, qui peut être considéré comme le masquage d'une obligation juridique par une forme de générosité et de sollicitude, selon une logique du don voulant contrebalancer la logique du droit. On peut y retrouver la provenance idéelle chrétienne, relevée à propos des théologiens, et par Joan Le Goff (2010). Denis Segrestin (1985) souligne, de son côté, le retour du phénomène corporatiste. Lidée communautaire est aujourd'hui réanimée par la communication d'entreprise, qui prône les valeurs d'appartenance et de communauté d'intérêt.

Mais, communautarisme d'entreprise et contractualisme représentent deux cultures opposées, une culture du consensus contre une culture de la négociation, des coutumes institués contre un droit codifié, une communication privilégiant la fonction phatique ${ }^{5}$, le contact, la connexion psychologique contre une communication privilégiant l'information autour du contrat. "Dans une perspective contractuelle, l'appartenance de l'individu au groupe est négociée; dans une perspective communautaire, cette appartenance est originelle. " (d'Almeida, [2001] : 212). Nous retrouvons ici une pensée proche de celle d'Aristote, de Saint Thomas d'Aquin et des scholiastes, qui ne faisaient pas reposer la société médiévale sur le pacte mutuel, mais sur une nécessité de la vie en commun, comme nécessité de la vie humaine. Dans la perspective contractuelle, l'appartenance est à construire. Dans la perspective communautaire, elle est donnée d'avance et présupposée. Il ne s'agit pas d'un communisme à construire, mais d'une communauté originelle, au sens chrétien du terme. Au droit naturel de la communauté, fondé sur la coutume et le statut, s'oppose le droit contractuel, qui prévaut actuellement dans la société, et qui est un système formel et rationnel destiné à créer une cohésion différente, réfléchie et non affective.

Dominique Bessire et Hervé Mesure (2009 : 34) rappellent que les discussions sur la nature et sur la place à accorder à la communauté, au communautarisme, sont aujourd'hui animées par des penseurs ${ }^{6}$ qui voient dans la notion de citoyen une conception rousseauiste trop abstraite ou universelle

$5 \mathrm{La}$ fonction phatique d'un message consiste à établir un contact, une connexion psychologique entre le destinateur et le destinataire, un canal physique, qui permet d'établir et de maintenir la communication. (Jakobson, $1963: 217$ ).

6 Cf. notamment Mesure et Renaut, 2002 ; Wivorka, 2001 ; Wuhl, 2001. 
de l'homme, ignorante de ses enracinements communautaires réels. Pour ces théoriciens, chaque personne est singulière et ses communautés d'appartenance contribuent à sa singularité. Nous y voyons apparaître un nominalisme, qui ne se situe pas très loin de celui de Pierre Abélard (1079-1142) pour qui toutes choses étaient singulières, cette influence nominaliste différant cependant du nominalisme radical de Guillaume d'Ockham (circa 1285-1349), qui concevait la société comme une collection d'individus liés par des ordinations et des pactes mutuels. À l'opposé de la perspective communautaire, la perspective contractuelle ne fait référence à aucune appartenance collective, elle suppose simplement un accord de volonté, un contrat entre des êtres indépendants et autonomes, au sens abstrait du Code Civil. Dominique Bessire et Hervé Mesure (2009 : 33) relèvent que les théories du contrat social, issues de l'œuvre de Jean-Jacques Rousseau, ont voulu fonder une société qui serait une association d'individus, définie hors de toute référence communautaire.

Nicole d'Almeida ([2001] : 212) observe que, construction historique récente dans le monde du travail, la notion de contrat a succédé à la perspective communautaire, mais que l'inspiration communautaire paraît loin d'être tarie ; au point que l'on a pu parler d'entreprise communauté contemporaine, marquée par une focalisation sur le client et sur le groupe social, dont les discours se recentrent sur les valeurs internes, les coutumes, le métier et ses traditions. En fait, on assiste au double mouvement paradoxal, selon lequel un effritement actuel du modèle communautaire peut être entrevu au profit du modèle contractuel, en même temps que réapparaissent, par contraste, la pérennité, la force et la continuité d'un idéal communautaire, qui survit aux transformations économiques et sociales et ne cesse de prendre des formes nouvelles. En sorte que, si l'idée originelle communautaire, stigmatisée par Aristote et les théologiens médiévaux, paraît en déclin, ceci n'est que relatif et toujours soumis à des rebondissements.

Le déclin du modèle communautaire, d'un point de vue organisationnel, est inversement proportionnel à sa force symbolique et à sa présence dans la communication des entreprises. Il n'est pas seulement un vestige du passé, il est l'idéal de certains dirigeants et de nombreux communicants. Se situant à l'opposé du modèle du contrat et de la négociation, il renoue avec une vision communautaire ancienne, qui continue d'animer les discours et les pratiques actuels de la communication interne et externe.

Ainsi, la communication interne des entreprises présente, par substitution, l'échange d'obligations juridiquement définies du contrat comme un engagement fait d'implications et d'adhésions, de devoirs, encastrés dans des rapports moraux ou psychologiques de confiance ; à l'image, nous dirions, des relations féodales qui, si elles étaient contractuelles, étaient aussi des relations d'homme à homme. Les valeurs de loyauté et d'engagement sont mises en avant gommant la problématique des droits ${ }^{7}$. Cet engagement prend

7 Cf. à ce sujet : Mitchell et O’Nell, 1998. 
la forme d'une valeur faite d'adhésion active et d'acceptation des règles d'une concurrence de plus en plus âpre. Cette tendance se développe à un moment où le droit du travail implose, révélant le caractère incomplet du contrat de travail.

Or, cette incomplétude laisse apparaître, justement, une perspective communautaire, en grande partie symbolique, faite de signes et de discours véhiculés par la communication de l'entreprise. En effet, au-delà de sa dimension gestionnaire, la communication institutionnelle de l'entreprise porte et construit un horizon communautaire. Nous pourrions presque dire par essence. En effet, elle fait le choix de l'appartenance et de l'union et transforme insensiblement le contrat en union, déplace le choix rationnel de la volonté contractante et lui substitue la force affective du lien. Elle enchante, en quelque sorte, le choix juridique, opère une alchimie juridique qui réunit deux options bien distinctes, contrat de travail et relation communautaire. La problématique communautaire s'inscrit, en fait, dans les insuffisances de la relation contractuelle, ou de sa métaphore, comme le souligne Nicole d'Almeida.

Pour Dominique Bessire et Hervé Mesure (2009 : 35), la communauté apparaît comme une des formes possibles de groupement humain, plus large que la famille, plus petite que la nation ou la société, différente du clan ou de la bande. Ce groupement est fondamentalement intermédiaire, puisqu'il englobe des groupements plus petits et s'insère dans d'autres plus larges. En second lieu, une communauté est faite des interactions entre ses membres. Ces interactions génèrent un nous, qui est plus que la somme des individus, et représente ce qui est commun entre les membres de la communauté. Toute communauté a donc des dimensions identitaires, qui se manifestent notamment dans le but et la mission qui l'animent, de manière explicite ou implicite. L'entreprise est ce lieu d'interactions qui donne naissance à une nouvelle identité, entendue ici comme un projet au service d'une mission ou going concern, au sens de John Rogers Commons ([1934]) $)^{8}$. Du point de vue communautaire, chaque entreprise est éminemment singulière, tout comme chaque communauté, nous dirions au sens nominaliste du terme. Une communauté peut aussi être définie quant au mode de vie, au patrimoine, à la culture, aux valeurs, au territoire, aux communautés de pratiques, etc. Ces communautés de pratiques apparaissent comme le lieu privilégié de la créativité, de l'émergence d'innovations. Or, la cohérence de la firme suppose la formation d'un sens et d'une vision commune qui guident des acteurs hétérogènes, en conciliant leurs intérêts antinomiques. L'entreprise, en tant que communauté, suppose donc une rupture avec le seul paradigme utilitariste, stigmatisé par Joan Le Goff et Pierre Legendre, puisque cela sous-entend aussi l'intégration dans des ensembles plus grands, jusqu'à en faire un des fondements de la RSE.

8 Cf. Bazzoli et Dutraive, 2002. 


\section{Conclusion}

Pour Philippe de Woot (1968), l'entreprise a une fin propre, distincte des individus qui la composent, mais aussi de l'intérêt général qui la dépasse. Ce n'est qu'en accomplissant sa fonction spécifique qu'elle contribue au bien commun et permet aux individus de réaliser leurs fins propres. Cette fonction spécifique, c'est la créativité économique. L'entreprise contribue ainsi au bien commun, car cette créativité constitue le ressort du progrès social et en fournit les moyens. Dominique Bessire et Hervé Mesure (2009 : 40) voient dans la définition de Philippe de Woot les trois éléments caractérisant les communautés de pratiques : une finalité qui transcende les intérêts de leurs membres, un lieu de créativité, une exigence de sens.

En tant que communauté, l'entreprise acquiert une dimension politique (Bessire, Mesure, 2009 : 33, 41). Le concept de communauté a, en effet, partie liée avec le politique au sens le plus large, qui remonte à Aristote, lequel concevait la communauté comme le cadre de vie naturel, dans lequel chacun peut s'accomplir, la cité apparaissant comme une méta-communauté, puisqu'elle englobe toutes les communautés. C'est le XVIII ${ }^{\mathrm{e}}$ siècle qui a induit la distinction entre les concepts de communauté et de société. La théorie rousseauiste du contrat social a fondé une société entendue, de façon abstraite, comme une association d'individus, définie hors de toute référence communautaire, l'individu devenant un citoyen. De ce fait, la conception rousseauiste a abouti à ce que le politique ne puisse se penser qu'à l'aide du triptyque théorique individu-citoyen, société, État, qui, dans ses extrêmes, a pu aboutir à un contractualisme exacerbé, empreint de surveillance, tout comme il a aussi rendu possible certaines formes de totalitarisme, tout dépendant alors de la place accordée à l'individu.

En sorte que le débat autour de la RSE laisse ressurgir la dialectique de très anciennes problématiques, mais aussi un retour à l'idée communautaire originelle. Encore faudrait-il qu'il s'agisse d'un sens communautaire bien senti, c'est-à-dire dans le respect de la liberté des membres de cette communauté. Il s'agit de questions primordiales que l'abstraction du contrat social rousseauiste n'a pu gommer, en tant que construction théorique ne pouvant englober tous les aspects du réel humain. Reste à préserver ces libertés auxquelles la RSE, parfois élevée au rang de mythe moderne par la communication, risquerait de porter atteinte, au nom d'une nécessité, vraie et justifiée, mais qu'il ne faudrait pas ériger en absolu ou en utopie, au détriment d'autres valeurs, comme le stigmatise Joan Le Goff. Tout étant question de mesure. 


\section{BIBLIOGRAPHIG}

ALMEIDA (d') Nicole, Les promesses de la communication [2001], Paris : Sciences modernités philosophie, PUF, 2007.

BASCHET Jérôme, La civilisation féodale: De l'an mil à la colonisation de l'Amérique, Paris : Champs, Flammarion, 2006, p. 198-199.

BAZZOLI Laure, Dutraive Véronique, «L'entreprise comme organisation et comme institution : Un regard à partir de l'institutionnalisme de J. R. Commons ", Économie et institutions, 2002/2, n ${ }^{\circ}$, p. 5-46.

BEC Christian, «Sur l'historiographie marchande à Florence au XIV e siècle », Daniel Poirion (Dir.) La chronique et l'histoire au Moyen Âge, PU Paris-Sorbonne, 1984, p. 46-52.

BESSIRE Dominique, MESURE Hervé, « Penser l'entreprise comme communauté : Fondements, définition et implications ", in Management $\&$ Avenir, 2009/10, n ${ }^{\circ} 30$, p. $30-50$.

BLOCH Marc, "Féodalité, vassalité, seigneurie : à propos de quelques travaux récents ", Annales d'histoire économique et sociale, 1931, n 10, p. 246-260.

BRAID Robert, «Justice, prix, salaires : Les théories médiévales de la gouvernance économique ", Justice and Economics : ancient doctrines and modern theories, Actes de la conférence, Université Toulouse I Capitole, 16-17 Juin 2011.

BRANTS Victor, «Fragments d'économie politique du Moyen Âge », Revue NéoScolastique, 1895, n $^{\circ} 5$, p. 27-48.

BRAUDEL Fernand, Civilisation matérielle, économie et capitalisme $X V^{E}-X V I I I^{e}$ siècle : Tome 2 : Les jeux de l'échange, Paris : Armand Colin, 1979.

COMMONS John Rogers, Institutional Economics : Its place in Political Economy [1934], New Brunswick : MacMillan, Transaction publishers, 1990.

COURTEMANCHE Danièle, Chopin-Pagotto Myriam, "Mémoire urbaine et biographie dans deux chroniques urbaines du XVI ${ }^{\mathrm{e}}$ siècle », in Elizabeth Gaucher, Aimé Petit (Dir.), Bien Dire et Bien Aprandre, 2002, n 20, p. 39-51.

FOVIAUX Jacques, De l'empire romain à la féodalité : Droit et institutions. Tome 1, Paris : Economica, 1986.

FREEMAN R. Edward, Strategic management: A stakeholder Approach, Boston, Pitman, 1984.

HABERMAS Jürgen, L'espace public : Archéologie de la publicité comme dimension constitutive de la société bourgeoise [1962], titre original Strukturwandel der Öffentlichkeit : Untersuchungen zu einer Kategorie der bürgerlichen Gesellschaft [1962], Paris : Critique de la politique, Payot, 1997.

HABIB Laurent, La communication performative: Pour en finir avec les idées vaines, Paris : PUF, 2010.

HEERS Jacques, La ville au Moyen âge en Occident: Paysages, pouvoirs et conflits [1990], Paris : Pluriel, L'Histoire en revue, Hachette littérature, 2004.

JAKOBSON Roman, Essais de linguistique générale : Tome 1, Paris, Minuit, 1963.

JORDA Henri, Le Moyen Agge des marchands : L'utile et le nécessaire, Paris : Krisis, Economie et Innovation, Innoval, L'Harmattan, 2002. 
LE GOFF Jacques, Le Moyen Âge et l'argent : Essai d'anthropologie historique, Paris : Perrin, 2010.

LE GOFF Jacques, Marchands et banquiers du Moyen Âge [1956], Paris : Que sais-je ? PUF, n699, 1993.

LE GOFF Joan, « La doctrine de la RSE est-elle socialement responsable ? », ESKA, Revue internationale de psychosociologie et de gestion des comportements organisationnels, 2010/38, Vol. XVI, p. 275-291.

LEGENDRE Pierre, Leçons IX : L'autre Bible de l'Occident : Le monument romanocanonique : Étude sur l'architecture dogmatique des sociétés, Paris : Fayard, 2009.

LEGENDRE Pierre, Leçons VII : Le désir politique de Dieu : Études sur les montages de l'État du Droit, Paris : Fayard, 2005.

LEGENDRE Pierre, Sur la question dogmatique en Occident : Aspects théoriques, Paris : Fayard, 1999.

LEGENDRE Pierre, Paroles poétiques échappées du texte : Leçons sur la communication industrielle, Paris : Seuil, 1982.

LIBAERT Thierry, "L'évolution de la communication d'entreprise », Recherches en communication, 2006, $\mathrm{n}^{\circ}$ 25, p. 41-52.

MESURE Sylvie, Renaut Alain, Alter Ego : Les paradoxes de l'identité démocratique, Paris : Champs, Flammarion, 2002.

MITCHELL Ronald K., O’Nell Stephen J., « Feudal institutions and small business governance : An exploration and comparison ", Journal of Small Business and Enterprise Development, 1998/5, n 3, p. 208-218.

PERNOUD Régine, Histoire de la bourgeoisie en France : Tome 1, Paris : Points, Seuil, 1992.

POLANYI Karl, La grande transformation : Aux origines politiques et économiques de notre temps [1944], Paris : Gallimard, 1983.

POSTEL Nicolas, SOBEL Richard, «Polanyi contre Freeman », Revue de la régulation, 2011/1, n 9. http://regulation.revues.org/9187.

POSTEL Nicolas, SOBEL Richard, « La RSE : nouvelle forme de démarchandisation du monde ? Une lecture institutionnaliste à partir de Karl Polanyi ", Développement durable et territoires 2010/1, n ${ }^{\circ}$ 31. http://developpementdurable.revues.org/8506.

SEGRESTIN Denis, Le phénomène corporatiste : Essai sur l'avenir des systèmes professionnels fermés en France, Paris : Fondation Saint-Simon, Fayard, 1985.

SUPIOT Alain, Homo juridicus : Essai sur la fonction anthropologique du droit, Paris : Points, Essais, Seuil, 2009.

SUPIOT Alain, «Les deux visages de la contractualisation : déconstruction du droit et renaissance féodale ", Sandrine CHASSAGNARD-PINET, David HIEZ (Dir.), Approche critique de la contractualisation, Paris : Droit et Sociétés, LGDJ, 2007, p. 19-44.

SUPIOT Alain, "La contractualisation de la société ", in Conférence de la Mission 2000, CNAM, 22 Février 2000, article publié sur le blog de Michel Balat. http://www. balat.fr/La-contractualisation-de-la.html. 
VIERS Jacques, BRULOIS Vincent, « L'évidente interpellation de la sociologie par la RSE ", in Sociologies Pratiques, RSE : l'entreprise au défi de la responsabilité sociale, 2009/1, $\mathrm{n}^{\circ} 18$, p. 1-6.

WEBER Max, La ville, [1921], extrait du tome 2 d'Économie et société, Philippe Fritsch (trad.), Paris : Champ urbain, Aubier Montaigne, 1982.

WIVORKA Michel, La différence, Paris : Balland, 2001.

WOLTON Dominique, Sauver la communication, Paris : Champs, Flammarion, 2007. WOOT (de) Philippe, Pour une doctrine de l'entreprise, Paris : Seuil, 1968.

WUHL Simon, L'égalité : Nouveaux débats, Paris : PUF, 2001.

Résumé : Si nous examinons le discours de la responsabilité sociale, ou plus largement, sociétale des entreprises, plusieurs conceptions s'opposent : contractuelle, institutionnelle, communautaire. La dialectique de ces oppositions laisse apparaître de très anciennes problématiques, à la fois historiques et philosophiques, dépassant la notion elle-même. Cet article s'attache donc aux fondements de la RSE, afin de mieux comprendre son pourquoi, sa nécessité, mais aussi les risques et dérives potentiels intrinsèques au concept lui-même.

Mots-clés: Responsabilité sociétale des entreprises, RSE, communication institutionnelle, contrat, institution, communautaire.

Abstract: If we examine the corporate social responsibility concept, opposite ideas will be set
in contradiction : contractual arrangements, institutional links and community relationships.
The dialectic of these contradictory ideas reveal old historical and philosophical debatable
issues, transcending the concept itself. This article aims to study the roots of corporate social
responsibility, in order to a better understanding of this idea, its reasons and necessity, but also
its risks of potential drifts.

Keywords : Corporate social responsibility, CSR, corporate communication, contract, institution. 